\title{
The cosmic microwave background
}

Jean Michel Lamarre and Jean-Loup Puget

Institut d'Astrophysique Spatiale

Université Paris-Sud, F-91405 Orsay, France

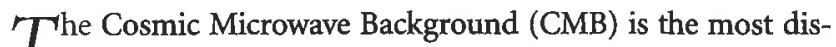

$I$ tant, and therefore the most ancient source of electromagnetic radiation that can be directly observed from Earth in any frequency range. The Cosmic Background Explorer satellite (COBE) has measured its sub-millimeter emission, which is that of a nearly perfect blackbody at $2.73 \mathrm{~K}$. The relative deviation from a pure Planck spectrum is very small (typically less than $10^{-5}$ ). This emission is attributed to the primordial Universe when it was about 300000 years old and warm enough $(3000 \mathrm{~K})$ to ionise the hydrogen gas that constitutes most of its mass. Owing to the expansion of the Universe, this radiation was red-shifted by the Doppler effect by a factor of about 1000, and thanks to the cooling due to the expansion, it could travel and reach us through the very transparent neutral hydrogen. The discovery of the CMB and even more the measurement of its Planck spectrum by the COBE-FIRAS ${ }^{1}$ experiment is the most compelling evidence for a hot big bang model. The existence of the CMB was predicted by Alpher, Bethe and Gamow as a very unique feature of a hot big bang in which the nucleosynthesis of most of the $\mathrm{He}, \mathrm{D}$ and ${ }^{7} \mathrm{Li}$ seen in the Universe (but which cannot be produced in stars) is produced in the early phases of a hot big bang.

\section{Anisotropies in the CMB - and what they tell us}

Furthermore, the existence of very small anisotropies was predicted at a level of $10^{-5}$ in the simplest model to explain large-scale structure formation in the Universe. In this model anisotropies result from the gravitational instability acting on in homogeneities generated in the very early Universe. These anisotropies were indeed found at the right level by the COBE$\mathrm{DMR}^{2}$ experiment.

In this model, these fluctuations carry information on the physics of the very early Universe because the spectrum of fluctuations is conserved at least on the largest scale in the expansion. The fluctuations on smaller scales are not gravitationally unstable during the whole history of the Universe preceding recombination. The fluctuations behave as acoustic oscillations leaving characteristic peaks of the anisotropies in the power spectrum. The lowest frequency acoustic peak corresponds to an angular scale, which depends mainly on the geometry of the spatial part of space-time. In fact, in the simplest model of structure formation, this power spectrum, if measures down to small enough scales and with enough accuracy, contains information allowing cosmologists to get very precisely all the cosmological parameters (space-time geometry, relative contribution of the various terms contributing to the dynamics of the Universe, etc.). The measurements of the small-scale anisotropies of the CMB are thus becoming one of the main tools of observational cosmology.

New results from the balloon-borne experiments BOOMERanG ${ }^{3}$ and MAXIMA ${ }^{4}$ and from ground-based experiments using radio detectors and interferometry $y^{5,6}$, were recently published. They gave a first view of the small-scale anisotropy of the $C M B$, unveiling the predicted peaks in the power spectrum of
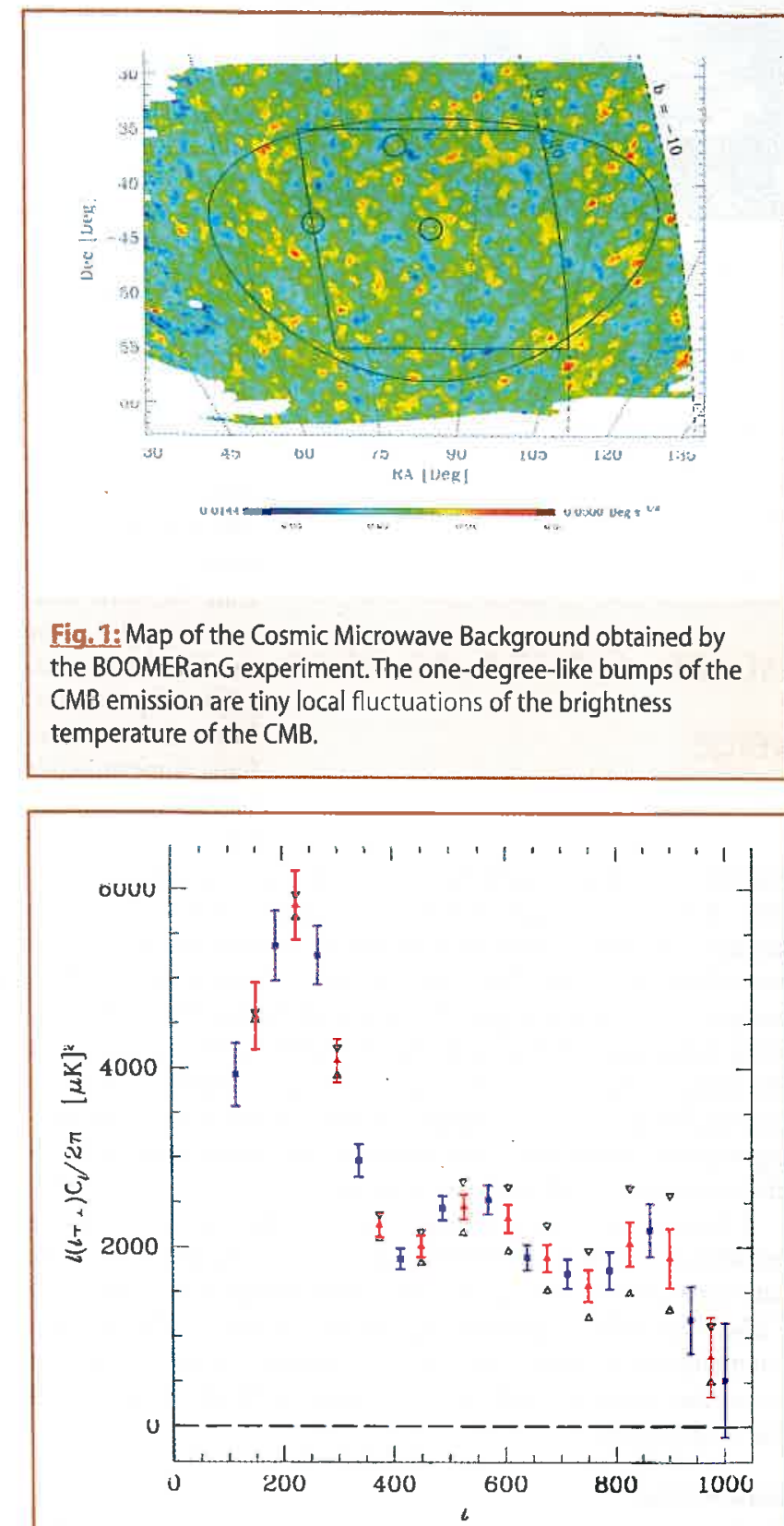

Fig. 2: The power spectrum of CMB anisotropies as seen by BOOMEranG. The first peak corresponds to an angular scale of about 1 degree as predicted for à "flat Universe" (i.e., a Universe where the spatial part of the metric is Euclidean). 
its angular distribution. The BOOMERanG $\mathrm{CMB}$ map shown in figure 1 is a high-signalto-noise rendition of the anisotropies of the background radiation as they emerged at recombination when the Universe was a billion times denser than at present. These anisotropies reflect the inhomogeneities of the early Universe. Figure 2 shows the combined power spectrum of these recent experiments. The predicted acoustic peaks are very clearly seen. The most striking result is that the position of the first one is within a few percent of the position predicted for a Universe with a Euclidean spatial part of the metric: "the Universe is flat"!

Following COBE, NASA launched in the summer of 2001 the MAP satellite, which will be a second-generation CMB space experiment. The detectors are passively, i.e., radiatively cooled radio-type receivers with sensitivity comparable to the balloon-borne bolometer experiments but with significantly better capabilities for large-scale measurements and control of systematics.

\section{The ultimate mission: ESA's 'Planck' satellite}

The 'Planck' project of the European Space Agency, to be launched in 2007, is intended to be the third generation of $\mathrm{CMB}$ space experiments, pushing to its limits the knowledge that will be retrieved from the CMB observation with unprecedented angular resolution and sensitivity.

The six spectral bands of the High Frequency Instrument (HFI) on 'Planck' cover the frequency range between 100 and $1000 \mathrm{GHz}$ with an angular resolution of about 5 arcmin. Its sensitivity will be limited, in the $\mathrm{CMB}$ channels, by the statistical fluctuations of the CMB itself, which makes 'Planck' a kind of ultimate experiment. It will also measure the polarization of the $\mathrm{CMB}$ in three channels, which will give independent and unique information on the $\mathrm{CMB}$ anisotropy.

This kind of accuracy on the CMB can be achieved only by removing the various astrophysical foregrounds emitting at these frequencies. Among these, one expects the emission of dust and gas in our own galaxy and from other galaxies. Clusters of galaxies, that contain high-temperature gas detected in the X-rays, distort the CMB spectrum by inverse Compton scattering. This is the Sunyaev-Zeldovich Effect (SZE), which makes clusters of galaxies good tracers of the dynamics of the Universe at large scales.

The six bands in HFI and four more in LFI (Low Frequency Instrument, also on 'Planck') are needed to characterise and eliminate these various components by use of their spectral and spatial signature. Planck must be considered not only as the third generation of CMB satellites, but also as the first sub-Terahertz all-sky survey of modern astronomy. Several thousands of galaxies, young stellar objects, clusters of galaxies will be detected, many of them for the first time. Nearly every field of astronomy will benefit from 'Planck's' results. The 'Planck' project is committed to deliver a set of well-defined products to the scientific community at large.

The extremely high sensitivity ( $\left.\mathrm{dT} / \mathrm{T}<210^{-6}\right)$ as well as the high angular resolution of 'Planck' made possible by the use of "Spider web" type bolometers developed at Caltech and flown on the BOOMERanG, MAXIMA and ARCHEOPS experiments. 'Planck' will also be the first experiment that measures the polarization signal of the $\mathrm{CMB}$ on large scales.
For a detailed discussion of all these questions we refer the reader to a more extensive review by Bouchet, Puget and Lamarre. $^{8}$

\section{References and Explanations of Abbreviations \\ ${ }^{1}$ Far Infrared Absolute Spectrophotometer \\ ${ }^{2}$ Differential Microwave Radiometers \\ ${ }^{3}$ P. de Bernardis et al. 2000, A flat universe from high resolution maps of the CMBR, Nature 404, 955.}

${ }^{4} \mathrm{~A}$. T. Lee et al, $A$ high spatial resolution analysis of the MAXIMA-I cosmic microwave background anisotropy data, astro-ph/0104459.

${ }^{5}$ A.D. Miller et al., A Measurement of the Angular Power Spectrum of the $C M B$ from $l=100$ to 400 , Accepted by Astrophys.J.Lett., Astro-ph/9906421.

${ }^{6} \mathrm{~N}$.W. Haverson et al., DASI first results: A Measurement of the Cosmic Microwave background Angular Power Spectrum, Astro-ph/0104489.

${ }^{7}$ Microwave Anisotropy Probe

${ }^{8}$ F.R. Bouchet, J.L. Puget, J.M. Lamarre 2000, The cosmic microwave background: from detector signals to constraints on the early universe physics, in 'The Primordial Universe', Binétruy et al., eds. (Les Ulis, Paris: EDP Sciences) pp. 103-220.

\section{About the authors}

Jean-Michel Lamarre, has 20 years of experience in IR and submm space astronomy. He contributed to the development of this field in France by leading or contributing to the conception of a number of experiments: EMILIE at the South Pole, the balloon-borne AROME experiment, and the space projects: CRYOSPIR, AELITA, SAMBA, FIRE and FIRST. He was the PI of the imaging channel of IKS on the VEGA sounder to the comet Halley, and of SPM-PRONAOS that measured the positive part of the SZ effect. He played a major role in the birth of the PlanckHFI concept and design, and is now the instrument scientist of this experiment.

Jean-Loup Puget is an astrophyicist and Research Director at CNRS. He is Mission Scientist for the ESA's Infrared Space Observatory (ISO), and also PI for the High Frequency Instrument (HFI) on ESA's Planck mission. In 1984, together with Alain Leger, he discovered the presence of large quantities of aromatic hydrocarbons in interstellar matter. 